\title{
Design of a Polarization-Independent Dual-Band Electromagnetically Induced Transparency-Like Metamaterial
}

\author{
Ozgur Demirkap ${ }^{1}$, Fulya Bagci ${ }^{*}$, A. Egemen Yilmaz ${ }^{2}$, Baris Akaoglu1 \\ ${ }^{1}$ Department of Physics Engineering, Ankara University, Ankara, Turkey \\ ${ }^{2}$ Department of Electrical and Electronics Engineering, Ankara University, Ankara, Turkey \\ *corresponding author, E-mail: Fulya.Bagcileng. ankara.edu.tr
}

\begin{abstract}
In this study, classical analogs of single and dual-band electromagnetically induced transparency are demonstrated with a four-fold symmetric metamaterial consisting of a Minkowski fractal ring resonator surrounded by a square ring resonator. The proposed metamaterials show high transmission ratios at the polarization independent resonances, as confirmed by the applied two different numerical methods. The proposed first and second metamaterials show electromagnetically induced transparency-like peaks at $8.87 \mathrm{GHz}$ and $9.30 \mathrm{GHz}$ with $93.2 \%$ and $93.1 \%$ transmission ratios, respectively. The two metamaterials are deliberately arranged in a $2 \times 2$ supercell array to yield a polarization independent dual-band electromagnetically induced transparency-like metamaterial. As a result, two transmission peaks emerge at 8.77 $\mathrm{GHz}$ and $9.22 \mathrm{GHz}$ with $81.3 \%$ and $90.7 \%$ transmission ratios, respectively. Delay-bandwidth products, which are regarded as a measure of slow-light ability, are found to be 0.34 and 0.61 at the resonances of the dual-band metamaterial. The peak frequencies and transmission ratios maintain not only for different polarization angles but also for oblique angles of incidence. These features of the proposed metamaterials are promising for single and multi-band filtering applications, phaseshifters, as well as for slow light and sensing devices.
\end{abstract}

\section{Introduction}

Metamaterials are sub-wavelength periodic structures which can be employed to produce unusual electromagnetic properties, such as negative refraction, super-lensing, nearunity absorption [1-3]. The design flexibility of metamaterials allows them to be used in a variety of applications in the fields of optics, electronics and as $\mathrm{RF} /$ microwave components. Distinctive properties of metamaterials have been exploited in a variety of emerging application fields, such as in energy harvesting [4-6], antenna technologies [8-14], biosensing [15-17] and slowlight devices $[18,19]$.

In recent years, low-loss substrates are used in metamaterial absorbers to capture the electromagnetic energy and convert to DC voltage using Schottky diodes [4], PIN diodes [5] or rectifier circuits [6]. In antenna technologies, metamaterials are used to increase the impedance bandwidth [7], reduce the size and extend the operation bandwidth $[8,9]$, enhance the gain and radiation efficiency [10-12] and to widen the angle of beam steering [13]. For mass production, the uses of lumped components, via-holes and defected ground structures are avoided by using metamaterials or metamaterial based transmission lines in antennas [14]. In the field of biosensing, small number of molecules in dilute solutions can be detected, thanks to label-free biosensors based on metamaterials [15]. Resonant transmission or reflection features of metamaterials lead to very sharp transmission or reflection peaks, which are remarkably desirable for developing highly sensitive sensors $[16,17]$. In addition, sharp peaks enable slow-light property so that the speed of electromagnetic waves can be manipulated in a way much more effectively than using cold atom-methods [18, 19].

One of the convenient methods for achieving slow-light propagation and electromagnetic wave control is the generation of electromagnetically induced transparency-like effect [20]. Although originally related to a quantum mechanical effect [21], the classical analogue of electromagnetically induced transparency has been observed in metamaterials as a result of destructive interference between different kinds of modes [22]. As a result of EITlike effect, a sharp transparency window appears inside a broad absorption band. The sharp transmission band yields a reduction in the group velocity of electromagnetic waves, which has many important applications in slow light devices, as well as in nonlinear optical and sensing applications [23, 24].

Classical analogues of EIT in metamaterials have been reported based on near-field coupling between bright and dark modes, bright and quasi-dark modes and bright and bright modes [25-29]. The bright mode can be directly excited by the incident electromagnetic waves, whereas the dark mode can only be excited by coupling with the bright mode. In this context, many different EIT-like metamaterials have been designed in microwave $[20,22$, $25,27-29]$, terahertz [23, 24, 26, 30] and optical frequency ranges [31]. However, most of the reported EIT-like metamaterials are sensitive to a fixed polarization of the impinging wave and have single-band of operation. On the other hand, many circular waveguide systems, fiber optic, radio-frequency and microwave communication systems work with circular or elliptical polarized waves. Therefore, polarization independent EIT-like metamaterials are very convenient to be used in slow-light devices, microwave 
phase shifters and filters.

In this study, we have designed, fabricated and investigated a dual-band polarization independent EIT-like metamaterial at microwave frequencies by using supercell method. In this method, two polarization independent single band EIT-like metamaterials are first designed to operate at the same resonance frequency. The unit cells of the designed EIT-like metamaterials are combined in a supercell configuration with $\mathrm{C}_{4}$ symmetry to create a dualband polarization independent EIT-like metamaterial. The proposed dual-band and polarization independent EIT-like metamaterial has potential to be employed in multi-band slow light devices, multi-frequency filtering, multi-mode phase shifters and in highly sensitive sensors.

\section{Design and modeling}

A square ring (SR) resonator and a first-order Minkowski fractal ring (MFR) resonator are used as the resonators of the unit cell. The resonators are made of copper $(35 \mu \mathrm{m}-$ thick) with a four-fold rotational symmetry to enable polarization independence. The unit cell constant, $p$, is selected to be $10 \mathrm{~mm}$ and the geometrical parameters of the two resonators are arranged to have a reflection resonance at close frequencies. Rogers RT Duroid/5880 substrate with a thickness of $1.575 \mathrm{~mm}$ and a dielectric constant of 2.2 is used as the substrate of the investigated metamaterials.

The magnitude and phase of transmission are calculated by two commercially available full-wave electromagnetic solvers to support the accuracy of the results. For this reason, a finite-integration based electromagnetic solver, CST Microwave Studio, and a finite-element method solver, Ansys HFSS, are used. The electromagnetic waves are sent to the structure along $z$-direction and, unit cell and master/slave boundaries are used during the calculations with CST and HFSS, respectively.

\section{Discussion}

The unit cell of the first EIT-like metamaterial is depicted schematically in Figure 1(a). The coupling distance between the two resonators is set as $0.5 \mathrm{~mm}$ for the proposed first EIT-like metamaterial. The geometrical parameters of the unit cell given in Figure 1(a) are as follows: $l=7.5 \mathrm{~mm}$, $w=0.5 \mathrm{~mm}, l_{1}=1.95 \mathrm{~mm}, w_{1}=0.2 \mathrm{~mm}, c=1.5 \mathrm{~mm}, d=2.0 \mathrm{~mm}$. The quality-factor ( $Q$-factor) of the resonators determines how the resonators interact with the incident electromagnetic wave. The $Q$-factor can be calculated by the division of the EIT-like peak frequency with respect to the full-width at half-maximum bandwidth of the band. The SR resonator solely in the unit cell has a reflection resonance at $10.55 \mathrm{GHz}$ with a $Q$-factor of 2.4. On the other hand, reflection resonance appears at $10.40 \mathrm{GHz}$ for the MFR resonator with a $Q$-factor of 8.1 . Therefore, SR and MFR resonators can be regarded as bright and quasi-dark resonators, respectively.

The transmission spectra of the first EIT-like metamaterial are given in Figure 1(b) for $x$-polarized and $y$ polarized incidence. When the resonators are combined in the unit cell, a transmission peak appears at $8.87 \mathrm{GHz}$ with a full-width of half-maximum (FWHM) bandwidth of 1.05 $\mathrm{GHz}$ and $93.2 \%$ transmission, as calculated by CST. On the other hand, HFSS calculations result in a transmission peak at $8.83 \mathrm{GHz}$ with $1.02 \mathrm{GHz}$ FWHM bandwidth and $91.5 \%$ transmission. There is a very good agreement between the calculated transmission spectra by the two methods. The overlap of the spectra belonging to $x$-polarized and $y$ polarized incidence shows that the proposed first EIT-like metamaterial has polarization independent character.

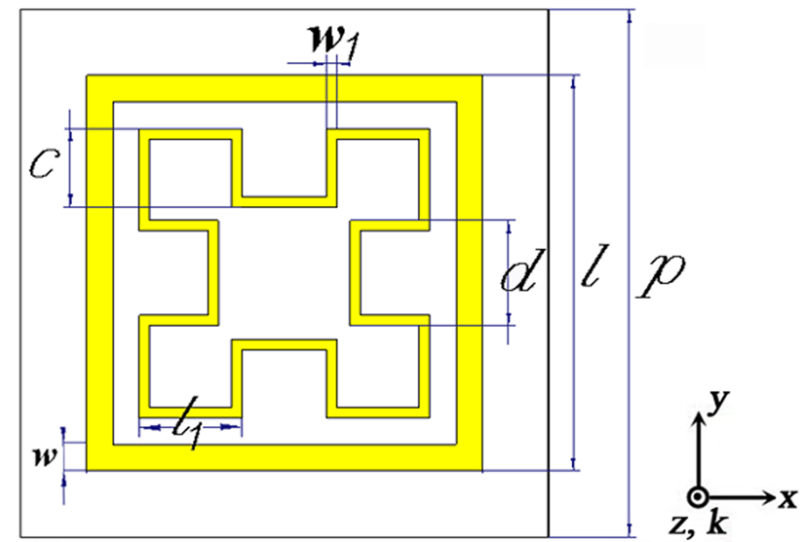

(a)
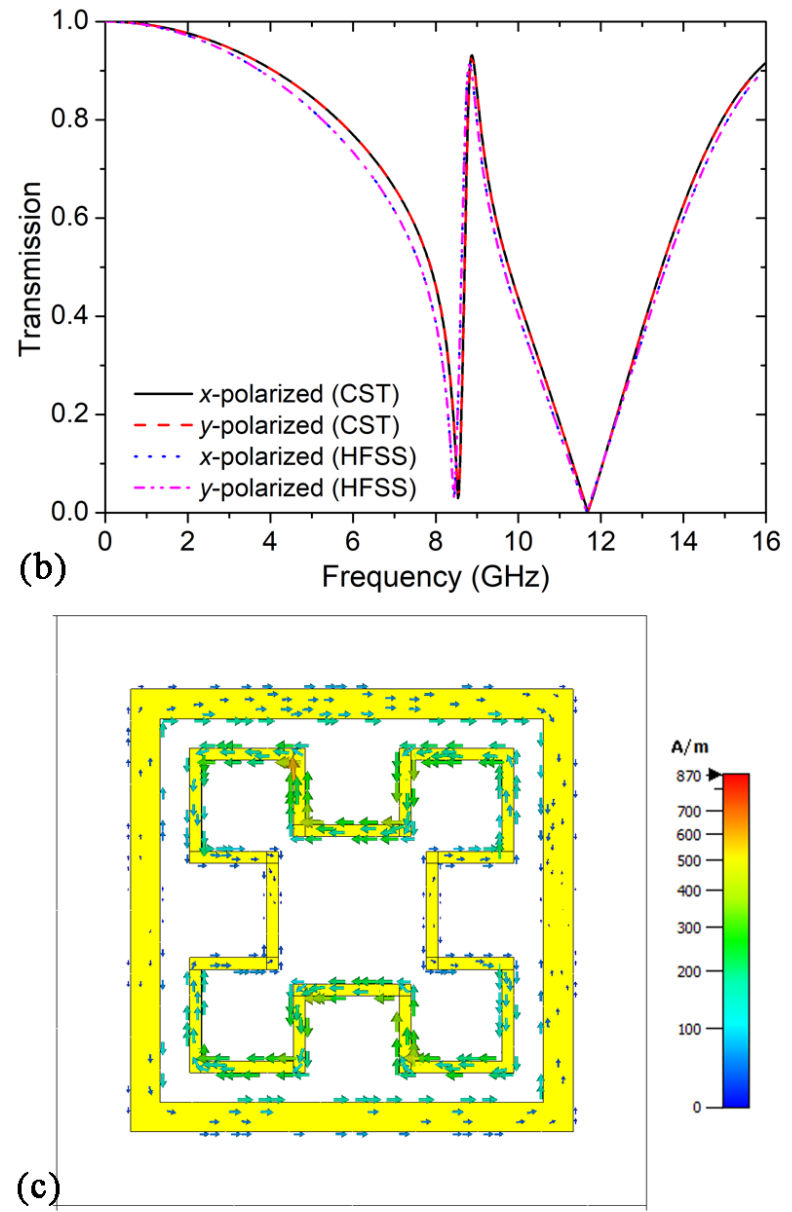

Figure 1: (a) Unit cell, (b) transmission spectra and (c) surface current distributions for the proposed first EIT-like metamaterial. 
The occurrence of the EIT-like effect can be confused with the Autler-Townes splitting (ATS)-like effect. It is demonstrated that ATS-like effect is active in strong coupling regime; whereas EIT-like effect is experienced in the weak coupling scheme $[32,33]$. In order to investigate if the coupling is weak or strong, surface current distributions are analyzed at the EIT-like peak frequency under $x$ polarization and are presented in Figure 1(c). Although SR is a bright resonator, surface currents accumulate much more in the MFR resonator, which is a quasi-dark resonator. This shows that near-field coupling between the SR and MFR resonators takes place. The amplitude of surface currents in the MFR resonator is moderate. This shows that the coupling between the resonators is not strong, indicating the occurrence of EIT-like effect.

In order to create the dual-band EIT-like metamaterial, a second EIT-like metamaterial with a transmission resonance at a close frequency to that of the first EIT-like metamaterial is aimed to be designed. The unit cell of the proposed second EIT-like metamaterial is schematically illustrated at Figure 2(a). The first step to design the EITlike metamaterial is to design the individual resonators to have a reflection resonance at the same frequency. The geometrical dimensions of the second EIT-like metamaterial are arranged as follows: $l=8.0 \mathrm{~mm}, w=0.8$ $\mathrm{mm}, l_{1}=1.95 \mathrm{~mm}, w_{1}=0.2 \mathrm{~mm}, c=1.7 \mathrm{~mm}$ and $d=1.5 \mathrm{~mm}$. There is a distance of $0.7 \mathrm{~mm}$ between the SR and MFR resonators.

The SR resonator solely has a reflection resonance at $10.49 \mathrm{GHz}$ with a $Q$-factor of 1.89 . On the other hand, MFR resonator has a reflection resonance at $10.65 \mathrm{GHz}$ with a $Q$ factor of 10.87 . When the two resonators are combined in one unit cell, a transparency window opens at $9.30 \mathrm{GHz}$ with a FWHM of $0.87 \mathrm{GHz}$ and a transparency of $93.1 \%$ (CST calculations). On the other hand, HFSS calculations point out a reflection resonance at $9.09 \mathrm{GHz}$ with a FWHM bandwidth of $0.87 \mathrm{GHz}$ and a transparency of $93.4 \%$. The transmission curves calculated by CST and HFSS are almost identical and indicate a polarization independent behavior, as demonstrated in Figure 2(b).

In order to explore the physical origin of EIT-like effect, surface current distributions are analyzed in Fig. 2(c) at the EIT-like peak frequency. It is clearly seen that surface currents hardly accumulate at the SR resonator; whereas the accumulation of surface currents in the MFR resonator is moderate so that there is a clear evidence for the EIT-like effect. The surface currents flow in opposite directions between the inner edge of the SR resonator and MFR resonator, which is a result of destructive interference between the two resonators. Hence, the net residual current in the SR resonator is very low so that radiation losses are diminished at the EIT-like peak frequency.

Two polarization independent single-band EIT-like metamaterials with transparency ratios greater than $90 \%$ have been designed. Finally, a polarization independent multi-band EIT-like metamaterial is formed by combining the designed two EIT-like metamaterials in a $2 \times 2$ supercell configuration with central symmetry. It would be beneficial to note here that the reason for choosing a $2 \times 2$ supercell rather that $1 \times 2$ or $2 \times 1$ is due to provide central symmetry for polarization independency. The schematic view of the designed dual-band EIT-like metamaterial is depicted in Figure 3(a) and the corresponding transmission spectra are plotted in Figure 3(b).

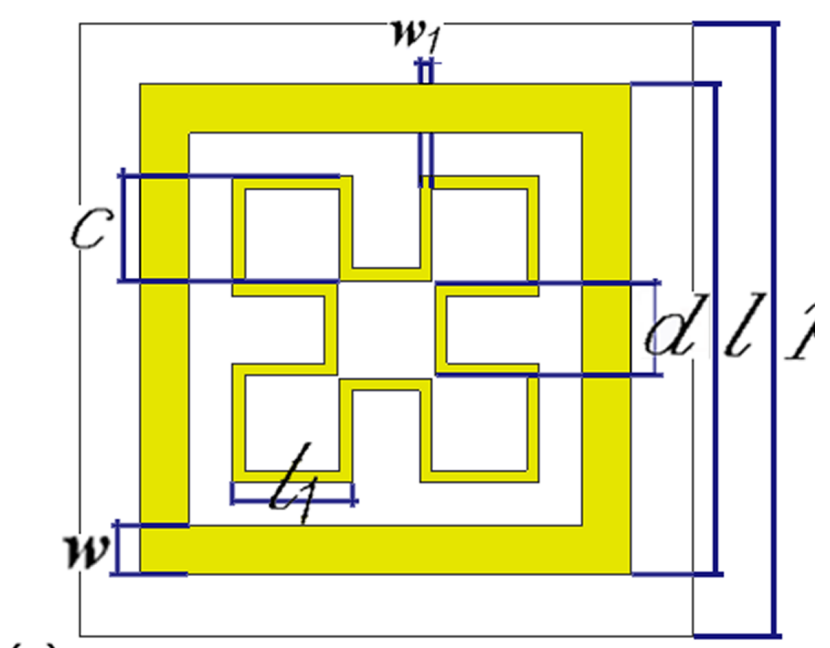

(a)

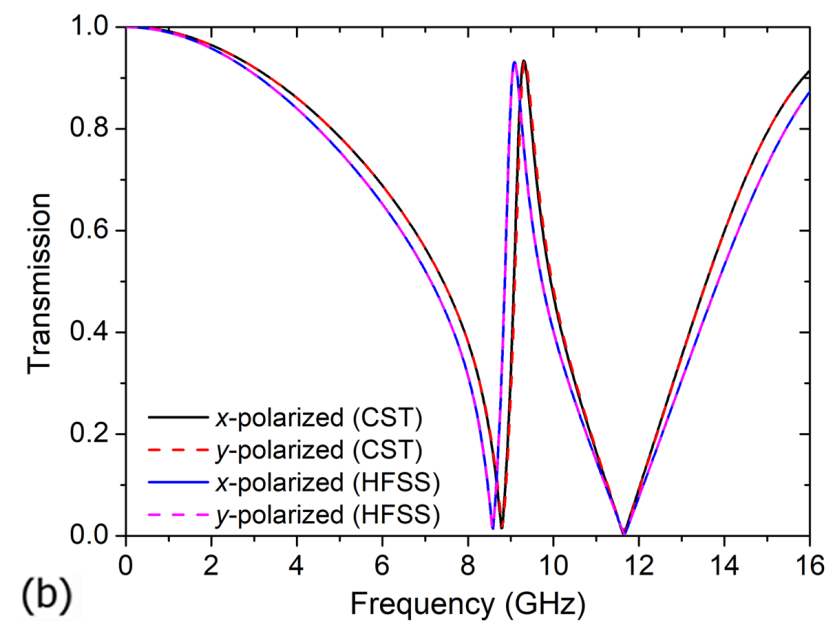

(c)

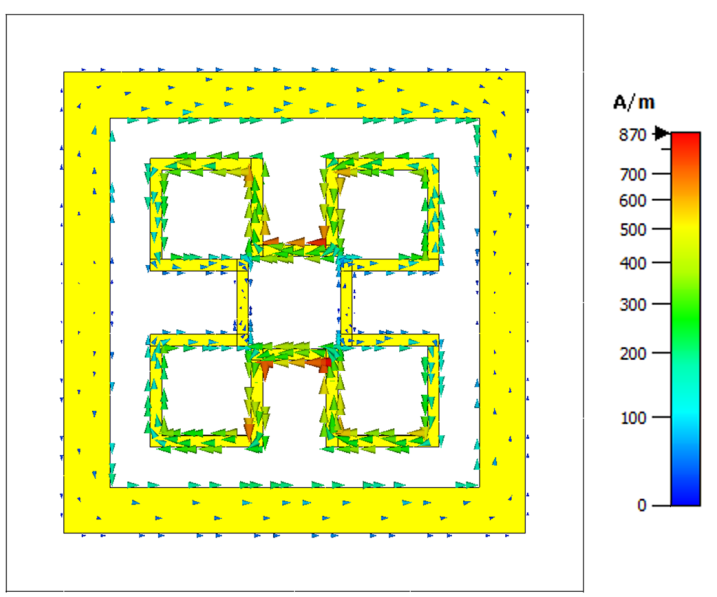

Figure 2: (a) Unit cell, (b) transmission spectra and (c) surface current distributions for the proposed second EITlike metamaterial. 
Two transmission bands emerge in a broad stop band between two transmission dips of $8.54 \mathrm{GHz}$ and $11.68 \mathrm{GHz}$, as a consequence of electromagnetic near-field coupling in the individual unit cells. Transmission peaks are found to be at $8.77 \mathrm{GHz}$ and $9.22 \mathrm{GHz}$ with transmission ratios of $81.3 \%$ and $90.7 \%$ and FWHM bandwidths of $0.20 \mathrm{GHz}$ and $0.74 \mathrm{GHz}$, respectively, from the calculations by CST. Besides, HFSS calculations also result in two transmission peaks at $8.23 \mathrm{GHz}$ and $9.07 \mathrm{GHz}$ with transmission ratios of $81.5 \%$ and $90.0 \%$ and FWHM bandwidths of $0.30 \mathrm{GHz}$ and $0.69 \mathrm{GHz}$, respectively. There is a very good agreement between the transmission results obtained from CST and HFSS and the proposed dual-band EIT-like metamaterial shows a polarization independent behaviour.
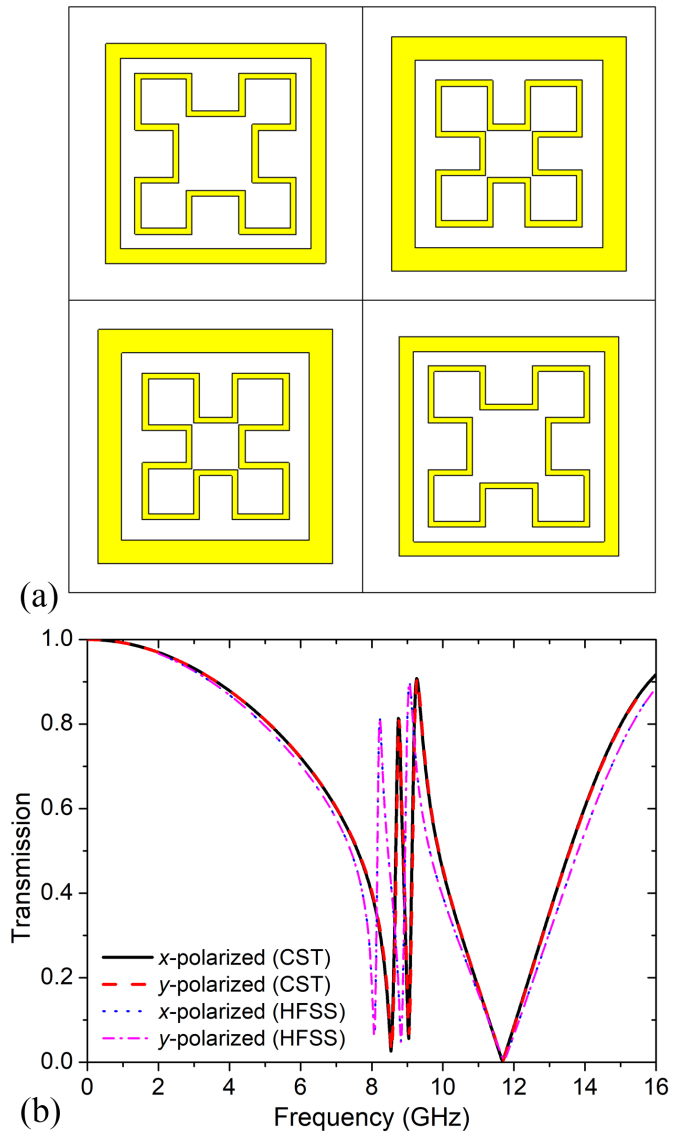

Figure 3: (a) Unit cell and (b) transmission spectra of the proposed dual-band polarization independent EIT-like metamaterial.

The most important outcome of electromagneticallyinduced transparency-like effect is the increase of the group delay of the electromagnetic waves while transmitting through the metamaterial sample. The group delay can be calculated from the transmission phase $(\Phi)$ data,

$$
\tau_{G}=-\frac{d \Phi}{d w}
$$

where $w$ is the angular frequency.
It would be worthwhile to associate the EIT-like peak resonances with the first or second EIT-like metamaterial as studied above. For this reason, surface current distributions are investigated by CST at the EIT-like resonances of 8.77 $\mathrm{GHz}$ and $9.22 \mathrm{GHz}$ and the corresponding results are presented in Figure 4. It is clear from Figure 4 that the first investigated EIT-like metamaterial is responsible for the existence of the first EIT-like peak at $8.77 \mathrm{GHz}$ and the second investigated EIT-like metamaterial is accounted for the second EIT-like peak at $9.22 \mathrm{GHz}$. It can also be deduced from the plot that there is not any electromagnetic near-field coupling between adjacent unit cells. The two EIT-like bands emerge as a consequence of near-field coupling between the resonators in different unit cells.
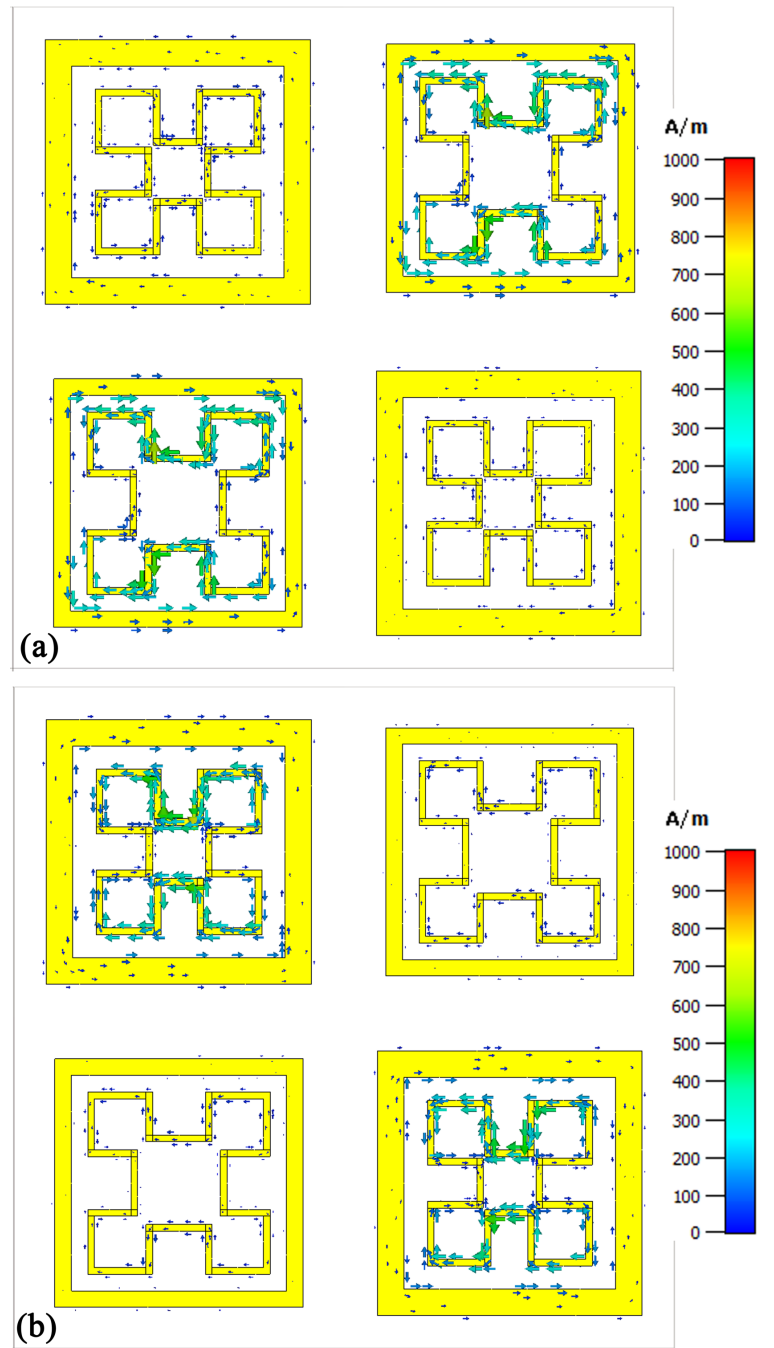

Figure 4: Surface current distributions of the dual-band EITlike metamaterial at the EIT-like resonances of (a) $8.77 \mathrm{GHz}$ and (b) $9.22 \mathrm{GHz}$.

The group delay spectrum is calculated from the wrapped transmission phase of CST. Since the transmission phase is completely identical for $x$-polarized and $y$-polarized incidence, the group delay spectrum is calculated for only one of the two orthogonal polarizations as shown in Figure 5. Electromagnetic waves delay by $1.72 \mathrm{~ns}$ and $0.82 \mathrm{~ns}$ at 
the two $S_{21}$ peak frequencies, $8.77 \mathrm{GHz}$ and $9.22 \mathrm{GHz}$, respectively. Group index can be calculated by the following formula $n_{\mathrm{G}}=(c / d) \tau_{\mathrm{G}}$, where $c$ is the speed of light in vacuum, $d$ is the thickness of the substrate and $\tau_{\mathrm{G}}$ is group delay $[29,34]$. Hence, group index is found to be 328 at $8.77 \mathrm{GHz}$ and 156 at $9.22 \mathrm{GHz}$. Delay-bandwidth product (DBP) is an important parameter to measure the ability of slow light for slow-wave filters and delay lines. It is calculated by the multiplication of group delay with FWHM bandwidth $[29,35,36]$. DBPs are found to be 0.34 and 0.61 for the dual-band EIT-like metamaterial at the peak transmission frequencies. The obtained DBP value of 0.61 is greater than those of single-band EIT-like metamaterials reported in $[29,35,36]$. A comparison of the transmission ratio, slow light capability and polarization dependency property of our dual-band EIT-like metamaterial with the literature is provided in Table 1. It can be clearly seen from the comparison that the proposed EIT-like metamaterial is novel due to having two EIT-like bands with high group index and transmission ratio values, as well as being polarization independent.

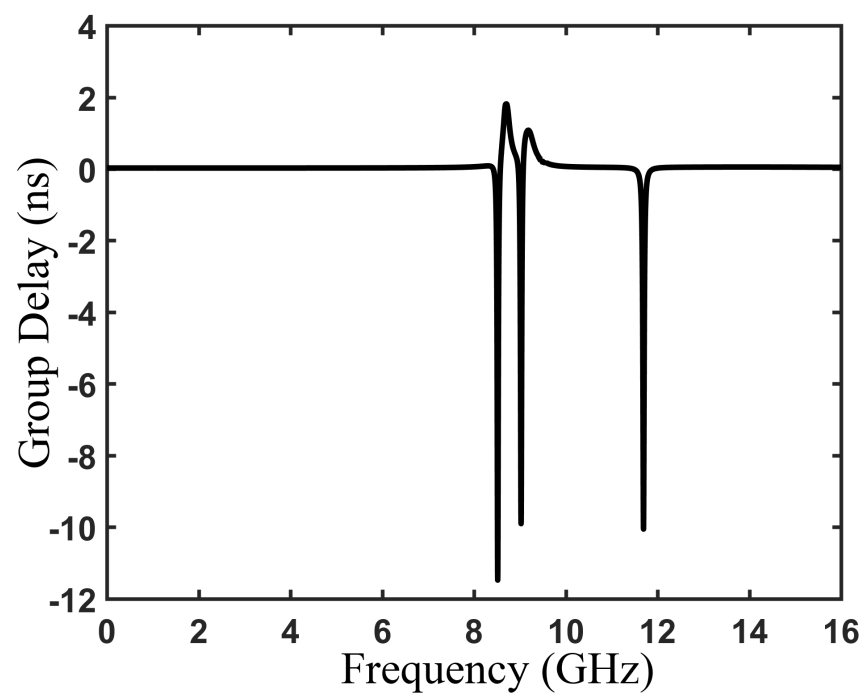

Figure 5: Group delay spectrum of the proposed dual-band polarization independent EIT-like metamaterial.

Table 1: Comparison of transmission, group index and polarization dependency properties of EIT-like metamaterials.

\begin{tabular}{cccc}
\hline Ref. & $\begin{array}{c}1^{\text {st }} \text { band } \\
\text { trans./group } \\
\text { index }\end{array}$ & $\begin{array}{c}2^{\text {nd }} \text { band } \\
\text { trans./group } \\
\text { index }\end{array}$ & $\begin{array}{c}\text { polarization } \\
\text { dependency }\end{array}$ \\
\hline$[29]$ & $83 \% / 103$ & - & independent \\
{$[35]$} & $>90 \% / 30$ & - & dependent \\
{$[34]$} & $71 \% / 150$ & - & dependent \\
{$[37]$} & $90 \% / 46$ & - & dependent \\
{$[38]$} & $60 \% / 185$ & - & independent \\
{$[39]$} & $-/ 125$ & - & independent \\
{$[40]$} & $63 \% / 247$ & $61 \% / 377$ & dependent \\
{$[41]$} & $92 \% /-$ & $88 \% /-$ & dependent \\
This & $81.3 \% / 328$ & $90.7 \% / 156$ & independent \\
\hline
\end{tabular}

It is desirable to keep the slow light properties constant for different oblique as well as polarization incidences for practical applications. Therefore, the dependence of the transmission spectra to various polarization angles is plotted in Figure 6. The proposed dual-band EIT-like metamaterial is completely polarization independent as it is apparent from Figure 6. Since the proposed metamaterial is designed to have a fourfold rotational symmetry, this is expected.

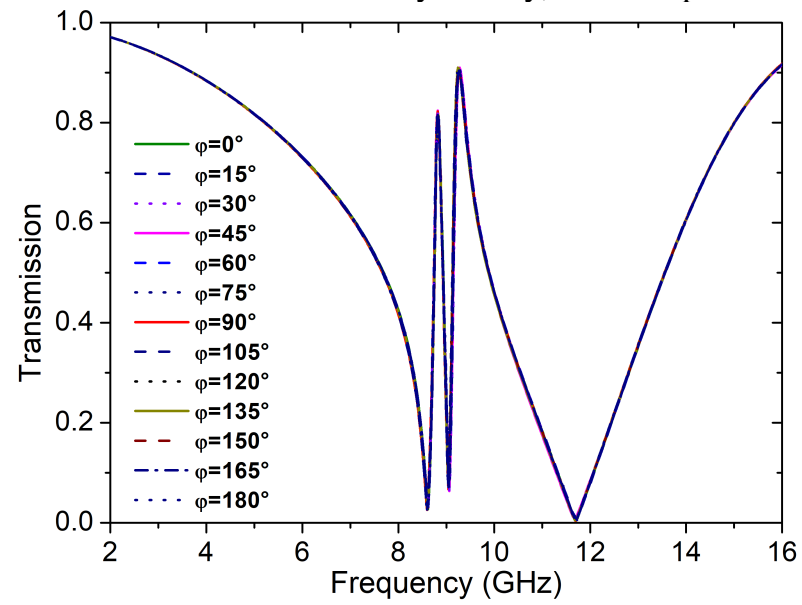

Figure 6: Transmission spectra of the proposed dual-band polarization independent EIT-like metamaterial for various polarization angles.

Transmission spectra of the proposed polarization independent EIT-like metamaterial under various oblique incidences are plotted in Figure 7 for $x$-polarized incidence and in Figure 8 for $y$-polarized incidence. Especially, the FWHM bandwidth of the second transmission band increases to $1.06 \mathrm{GHz}$ for $x$-polarized incidence, whereas it decreases to $0.46 \mathrm{GHz}$ for $y$-polarized incidence. The reason behind the increase (decrease) of bandwidth is the change of orientation of electric (magnetic) field vector as the incidence angle increases. On the other hand, EIT-like resonance frequencies unchange and transmission ratios slightly change, although the angle of incidence increases. This shows that the proposed polarization independent dualband EIT-like metamaterial shows a robust performance against the change of the incidence angle.

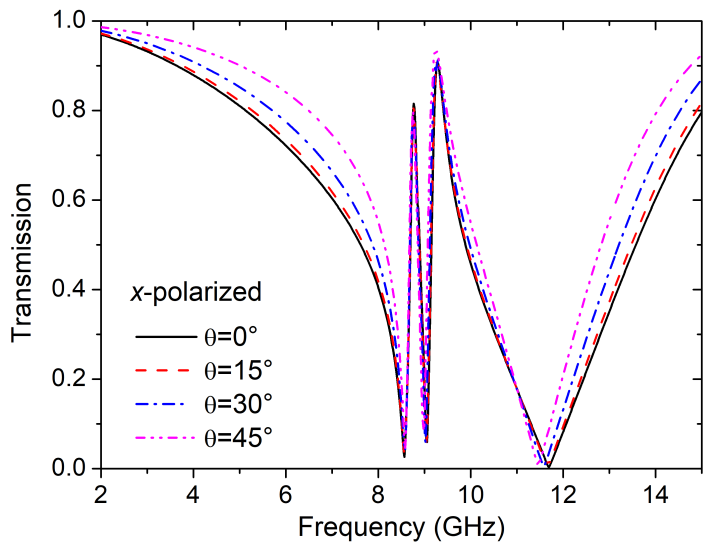

Figure 7: Transmission spectra of the proposed dual-band polarization independent EIT-like metamaterial for oblique angle of incidences under $x$-polarized incidence. 


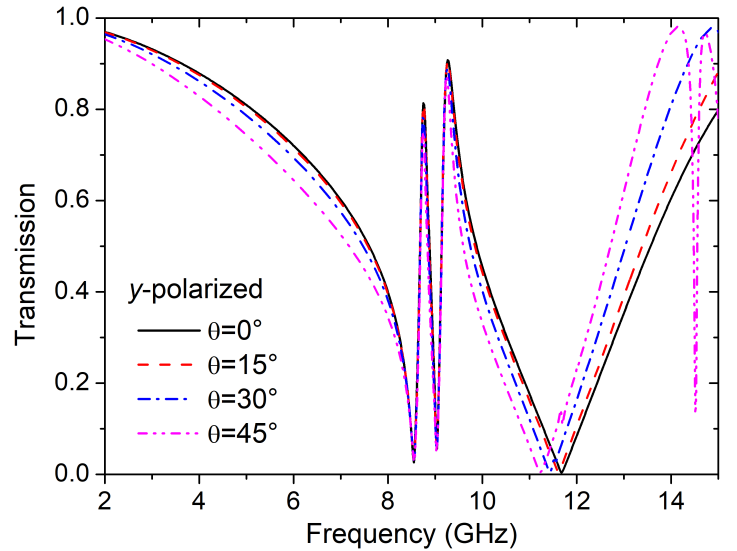

Figure 8: Transmission spectra of the proposed dual-band polarization independent EIT-like metamaterial for oblique angle of incidences under $x$-polarized incidence.

It is remarkable to note here that dual-band slow light effect is more demanding than single-band slow light effect, since it can be employed in multi-frequency filtering, multiband slow-light devices, multi-band phase shifters and in the development of more sensitive sensors [42-46]. The proposed dual-band EIT-like metamaterial has advantages in this respect and due to being polarization invariant it can be employed in applications unrestrictive from the direction of the impinging wave.

\section{Conclusions}

In this study, polarization independent, single and dual-band EIT-like metamaterials are designed by using two different numerical methods based on finite-integration technique and finite-element method. Both of the single and dual-band transmission peaks have more than $80 \%$ transmission. Delay-bandwidth products are calculated to be 0.34 and 0.61 for the dual-band EIT-like metamaterial. In addition to being completely polarization independent, the dual-band EIT-like metamaterial also exhibits a robust performance against the change of incidence angle.

\section{Acknowledgements}

This work is supported by The Scientific and Technological Research Council of Turkey (TUBITAK) under Grant No 117E504 and Ankara University Research Funds under Grant No 17B0443006.

\section{References}

[1] D. R. Smith, J. B. Pendry, M. C. K. Wiltshire, "Metamaterials and negative refractive index", Science 305(5685): 788-792, 2004.

[2] K. Aydin, I. Bulu, E. Ozbay, "Subwavelength resolution with a negative-index metamaterial superlens", Appl. Phys. Lett. 90: 254102, 2007.

[3] N. I. Landy, S. Sajuyigbe, J. J. Mock, D. R. Smith, W. J. Padilla, "Perfect metamaterial absorber", Phys. Rev. Lett. 100: 207402, 2008.
[4] A. M. Hawkes, A. R. Katko, S. A. Cummer, "A microwave metamaterial with integrated power harvesting functionality", Appl. Phys. Lett. 103: 163901, 2013.

[5] G. T. Oumbe Tekam, V. Ginis, J. Danckaert, P. Tassin, "Designing an efficient rectifying cut-wire metasurface for electromagnetic energy harvesting", Appl. Phys. Lett. 110: 083901, 2017.

[6] A. Z. Ashoor, O. M. Ramahi, "Polarization-independent cross-dipole energy harvesting surface", Trans. Microw. Theory Techn. 2019. doi:10.1109/TMTT.2018.2885754

[7] M. Alibakhshi-Kenari, B. S. Virdee, A. Ali, E. Limiti, "Extended aperture miniature antenna based on CRLH metamaterials for wireless communication systems operating over UHF to C-Band", Radio Sci. 53(2): 154 $165,2018$.

[8] M. Alibakhshi-Kenari, "Design and modeling of new UWB metamaterial planar cavity antennas with shrinking of the physical size for modern transceivers", Int. J. Antennas Propag. 2013: 562538, 2013.

[9] P. Dawar, N S Raghava, A. De, "Miniaturized UWB multi-resonance patch antenna loaded with novel modified H-shape SRR metamaterial for microspacecraft applications", Front. Inform. Tech. El. Eng. 18: 1883 -1891, 2017.

[10] M. Alibakhshi-Kenari, M. Naser-Moghadasi, R.A. Sadeghzadeh, B. S. Virdee, Ernesto Limiti, "Miniature CRLH-based ultra wideband antenna with gain enhancement for wireless communication applications", ICT Express 2(2): 75-79, 2016.

[11]M. Alibakhshi-Kenari, M. Naser-Moghadasi, R. Sadeghzadeh, "The resonating MTM based miniaturized antennas for wide-band RF-microwave systems" Microw. Opt. Techn. Let. 57(10): 2339-2344, 2015.

[12] M. Alibakhshi-Kenari, M. Naser-Moghadasi, R. A. Sadeghzadeh, B. S. Virdee, E. Limiti, "Traveling-wave antenna based on metamaterial transmission line structure for use in multiple wireless communication applications", AEU-Int J Electron C. 70(12): 16451650, 2016.

[13] M. Alibakhshikenari, B. S. Virdee, A. Ali, E. Limiti, “A novel monofilar-Archimedean metamaterial inspired leaky-wave antenna for scanning application for passive radar systems", Microw. Opt. Technol. Lett. 60: 20552060, 2018.

[14] M. Alibakhshikenari, B. S. Virdee, E. Limiti, "Compact single layer travelling-wave antenna design using metamaterial transmission-lines", Radio Sci. 52(12): 1510-1521, 2017.

[15] K. V. Sreekanth, Y. Alapan, M. ElKabbash, E. Ilker, M. Hinczewski, U. A. Gurkan, A. De Luca, G. Strangi, "Extreme sensitivity biosensing platform based on hyperbolic metamaterials", Nat. Mater. 15: 621-628, 2016.

[16] X.-J. He, L. Wang, J.-M. Wang, X.-H. Tian, J.-X. Jiang, Z.-X. Geng, "Electromagnetically induced transparency in planar complementary metamaterial for refractive 
index sensing applications", J. Phys. D: Appl. Phys. 46: 365302, 2013.

[17]Z. Vafapour, "Near infrared biosensor based on classical electromagnetically induced reflectance (CIEIR) in a planar complementary metamaterial", $O p t$ Commun. 387: 1-11, 2017.

[18] S. Yamasaki, T. Amemiya, Z. Gu, J. Suzuki, N. Nishiyama, S. Arai, "Analysis of the slow-light effect in silicon wire waveguides with metamaterials", J. Opt. Soc. Am. B. 35(4): 797-804, 2018.

[19] M. Parvinnezhad Hokmabadi, J.-H. Kim, E. Rivera, P. Kung, S. M. Kim, "Impact of substrate and bright resonances on group velocity in metamaterial without dark resonator", Sci. Rep. 5: 14373, 2015.

[20]N. Papasimakis, V. A. Fedotov, N. I. Zheludev, S. L. Prosvirnin, "Metamaterial analog of electromagnetically induced transparency", Phys. Rev. Lett. 101: 253903, 2008.

[21]S. E. Harris, "Electromagnetically induced transparency", Phys. Today 50(7): 36-42, 1997.

[22] C. Kurter, P. Tassin, L. Zhang, T. Koschny, A. P. Zhuravel, A. V. Ustinov, S. M. Anlage, C. M. Soukoulis, "Classical analogue of electromagnetically induced transparency with a metal-superconductor hybrid metamaterial", Phys. Rev. Lett. 107: 043901, 2011.

[23]Z. Vafapour, H. Alaei, "Achieving a high $Q$-factor and tunable slow-light via classical electromagnetically induced transparency (CI-EIT) in metamaterials", Plasmonics 12: 479-488, 2017.

[24]Z. G. Dong, H. Liu, J. X. Cao, T. Li, S. M. Wang, S. N. Zhu, X. Zhang, "Enhanced sensing performance by the plasmonic analog of electromagnetically induced transparency in active metamaterials", Appl. Phys. Lett. 97: 114101, 2010

[25]P. Tassin, L. Zhang, Th. Koschny, E. N. Economou, C. M. Soukoulis, "Planar designs for electromagnetically induced transparency in metamaterials", Opt. Express 17(7): 5595-5605, 2009.

[26] X. J. Liu, J. Q. Gu, R. Singh, Y. F. Ma, J. Zhu, Z. Tian, M. X. He, J. G. Han, W. L. Zhang, "Electromagnetically induced transparency in terahertz plasmonic metamaterials via dual excitation pathways of the dark mode", Appl. Phys. Lett. 100: 131101, 2012.

[27]F.-Y. Meng, Q. Wu, D. Erni, K. Wu, J.-C. Lee, "Polarization-independent metamaterial analog of electromagnetically induced transparency for a refractive-index-based sensor", IEEE Trans. Microwave Theory Tech. 60(10): 3013-3022, 2012.

[28] W. Wang, L. Zhang, K. Fang, Y. Zhang, "Experimental study of EIT-like phenomenon in a metamaterial plasma waveguide", AEM 1(3): $61-63,2012$.

[29]F. Bagci, B. Akaoglu, "A polarization independent electromagnetically induced transparency-like metamaterial with large group delay and delaybandwidth product", J. Appl. Phys. 123: 173101, 2018.

[30] M. P. Hokmabadi, J.-H. Kim, E. Rivera, P. Kung, S. M. $\mathrm{Kim}$, "Impact of substrate and bright resonances on group velocity in metamaterial without dark resonator", Sci. Rep. 5: 14373, 2015.

[31] X. R. Jin, J. Park, H. Zheng, S. Lee, Y. Lee, J. Y. Rhee, K. W. Kim, H. S. Cheong, and W. H. Jang, "Highly dispersive transparency at optical frequencies in planar metamaterials based on two-bright-mode coupling", Opt. Express 19(22): 21652-21657, 2011.

[32] L.-Y. He, T.-J. Wang, Y.-P. Gao, C. Cao, C. Wang, "Discerning electromagnetically induced transparency from Autler-Townes splitting in plasmonic waveguide and coupled resonators system", Opt. Express 23(18): 23817-23826, 2015.

[33]F. Nazari, M. Izadi, "Tunable Autler-Townes splitting using graphene-based electro-optic effect", Phys. Lett. A 382 (27): 1829-1834, 2018.

[34]F. Zhang, X. He, X. Zhou, Y. Zhou, S. An, G. Yu, and L. Pang, "Large group index induced by asymmetric split ring resonator dimer", Appl. Phys. Lett. 103: 221904, 2013.

[35] L. Zhang, P. Tassin, T. Koschny, C. Kurter, S. M. Anlage, C. M. Soukoulis, "Large group delay in a microwave metamaterial analogue of electromagnetically induced transparency", Appl. Phys. Lett. 97: 241904, 2010.

[36] H. Li, S. Liu, S. Liu, S. Wang, H. Zhang, B. Bian, X.-K. Kong, "Electromagnetically induced transparency with large delay-bandwidth product induced by magnetic resonance near field coupling to electric resonance", Appl. Phys. Lett. 106: 114101, 2015.

[37] L. Zhu, F.-Y. Meng, L. Dong, Q. Wu, B.-J. Che, J. Gao, J.-H. Fu, K. Zhang, and G.-H. Yang, "Magnetic metamaterial analog of electromagnetically induced transparency and absorption", J. Appl. Phys. 117: 17D146, 2015.

[38]H.-M. Li, F. Xue, "Tailoring polarization of electromagnetically induced transparency based on noncentrosymmetric metasurfaces", Phys. Lett. A 381: 3000-3004, 2017.

[39] L. Zhu, L. Dong, J. Guo, F. Y. Meng, X. J. He, T. H. $\mathrm{Wu}$, "Polarization-independent transparent effect in windmill-like metasurface", J. Phys. D: Appl. Phys. 51: 265101, 2018.

[40] K. Zhang, C. Wang, L. Qin, R.-W. Peng, D.-H. Xu, X. Xiong, M. Wang, "Dual-mode electromagnetically induced transparency and slow light in a terahertz metamaterial", Opt. Lett. 39(12): 3539-3542, 2014.

[41] S. Hu, H. Yang, S. Han, X. Huang, B. Xiao, “Tailoring dual-band electromagnetically induced transparency in planar metamaterials", J. Appl. Phys. 117: 043107, 2015.

[42]Z. Chen, Y. Wang, Y. Cheng, H. Yin, "Fast design and cold-circuit properties simulation for the slow wave structure of a $0.14 \mathrm{THz}$ broadband folded waveguide traveling wave tube", J. Infrared Millim. Terahertz Waves 31: 926-933, 2010.

[43]B. Lakshminarayanan, T. M. Weller, "Design and modeling of 4-bit slow-wave MEMS phase-shifters", IEEE Trans. Microw. Theory Tech. 54:120-127, 2006. 
[44]M. Amin, R. Ramzan, O. Siddiqui, "Slow wave applications of electromagnetically induced transparency in microstrip resonator", Sci. Rep. 8: 2357, 2018.

[45]B. Reinhard, K. M. Schmitt, V. Wollrab, J. Neu, R. Beigang, M. Rahm, "Metamaterial near-field sensor for deep-subwavelength thickness measurements and sensitive refractometry in the terahertz frequency range", Appl. Phys. Lett. 100: 221101, 2012.

[46] X. Q. Lin, Z. Chen, J. W. Yu, P. Q. Liu, P. F. Li, Z. D. Chen, "An EIT-based compact microwave sensor with double sensing functions", IEEE Sensors J. 16: 293298, 2016. 